Executive Editor: Altieres de Oliveira Silva Associate Editor: Manuel Portugal Ferreira, Ph.D.

Evaluation Process: Double Blind Review pelo SEER/OJS

\title{
O QUE SABEMOS SOBRE AS BARREIRAS À TRANSFERÊNCIA DE CONHECIMENTO? UM ESTUDO BIBLIOMÉTRICO SOBRE INTERNAL STICKINESS
}

\author{
${ }^{1}$ Valdemilson de Assis Alves de Araujo \\ ${ }^{2}$ Isabel Cristina Scafuto
}

\section{RESUMO}

Objetivo do estudo: identificar a estrutura intelectual da pesquisa sobre (IS) a partir das referências existentes nas publicações sobre esse tema.

Metodologia / Abordagem: foi usada uma análise bibliométrica de citação e cocitação, como também uma análise de rede. A amostra, após trabalhada e livre de artigos que não faziam parte do tema, foi composta por 152 artigos entre o ano de 1994 a 2018.

Originalidade / Relevância: A transferência de conhecimento e melhores práticas entre os membros da organização têm atraído considerações da academia e das organizações, porque a competitividade da empresa está diretamente ligada ao conhecimento adquirido e seu compartilhamento. A dificuldade de transferir conhecimento e melhores práticas nas organizações é representada pelo termo Internal Stickiness (IS).

Principais resultados: identificou-se três campos que direcionam as pesquisas sobre (IS): Transferência de Conhecimento; Transferência de Conhecimento como fator positivo para a organização; Transferência de Conhecimento e Inovação Estratégica.

Contribuições teóricas / metodológicas: foi possível identificar que não tinha nenhum artigo de revisão sobre o tema (IS). Então, este estudo pôde contribuir com a teoria e mostrar os trabalhos existentes que tiveram uma maior influência sobre (IS).

Contribuições sociais / para a gestão: percebe-se que os artigos existentes tratam de (IS) como a dificuldade de transferência de conhecimento. Com isto, este estudo pode contribuí também com a prática da transferência de conhecimento, principalmente, porque este tema está relacionado com o desenvolvimento da vantagem competitiva nas empresas.

Palavras-Chave: Internal Stickiness. Transferência de Conhecimento. Bibliométrico.

Received on September 11th, 2020 Approved on December 26th, 202

\section{(APA)}

Araujo, V., \& Scafuto, I. (2021). O que sabemos sobre as Barreiras à Transferência de Conhecimento? Um Estudo Bibliométrico sobre Internal Stickiness. International Journal of Professional Business Review, 6(1), 1-19. doi: http://dx.doi.org/10.26668/businessreview/2021.v6i1.212

\footnotetext{
${ }^{1}$ Mestre em Gestão de Projetos pelo Programa de Pós-graduação em Gestão de Projetos PPGP da Universidade Nove de Julho - UNINOVE, São Paulo, (Brasil). Gerente administrativo da Distribuidora de Papéis Eireli - DPN, São Paulo. E-mail: araujovaa.gp@gmail.com Orcid id: https://orcid.org/0000-0002-4976-6432

2 Doutora em Administração pela Universidade Nove de Julho - UNINOVE, São Paulo, (Brasil). Professora e Pesquisadora no Programa de Pós-Graduação em Gestão de Projetos - PPGP da Universidade Nove de Julho - UNINOVE. E-mail: isabelscafuto@gmail.com Orcid id: https://orcid.org/0000-0002-6788-3325
} 


\section{WHAT DO WE KNOW ABOUT BARRIERS TO KNOWLEDGE TRANSFER? A BIBLIOMETRIC STUDY ON INTERNAL STICKINESS}

\section{ABSTRACT}

Objective of the study: to identify the intellectual structure of research on (IS) from the references existing in publications on this topic.

Methodology / Approach: a bibliometric analysis of quotation and quotation was used, as well as a network analysis. The sample, after worked and free of articles that were not part of the theme, was composed of 152 articles from 1994 to 2018.

Originality / Relevance: The transfer of knowledge and best practices among members of the organization has attracted considerations from academia and organizations, because the company's competitiveness is directly linked to the knowledge acquired and its sharing. The difficulty of transferring knowledge and best practices in organizations is represented by the term Internal Stickiness (IS).

Main results: three fields were identified that guide research on (IS): Knowledge Transfer; Knowledge transfer as a positive factor for the organization; Knowledge Transfer and Strategic Innovation.

Theoretical / methodological contributions: it was possible to identify that there was no review article on the topic (IS). So, this study was able to contribute with the theory and show the existing works that had a greater influence on (IS).

Social / management contributions: it is noticed that the existing articles deal with (IS) as the difficulty of knowledge transfer. With this, this study can also contribute to the practice of knowledge transfer, mainly because this theme is related to the development of competitive advantage in companies.

Keywords: Internal Stickiness. Knowledge Transfer. Bibliometric. 


\section{¿QUÉ SABEMOS SOBRE LAS BARRERAS PARA LA TRANSFERENCIA DE CONOCIMIENTOS? UN ESTUDIO BIBLIOMÉTRICO SOBRE INTERNAL STICKINESS}

\section{RESUMEN}

Objetivo del estudio: identificar la estructura intelectual de la investigación sobre (IS) a partir de las referencias existentes en las publicaciones sobre este tema.

Metodología / Enfoque: se utilizó un análisis bibliométrico de cotización y cotización, así como un análisis de redes. La muestra, luego de trabajada y libre de artículos que no formaban parte de la temática, estuvo compuesta por 152 artículos de 1994 a 2018.

Originalidad / Relevancia: La transferencia de conocimiento y mejores prácticas entre los miembros de la organización ha atraído la consideración de la academia y las organizaciones, porque la competitividad de la empresa está directamente ligada al conocimiento adquirido y su intercambio. La dificultad de transferir conocimientos y mejores prácticas en las organizaciones está representada por el término Internal Stickiness (IS).

Resultados principales: se identificaron tres campos que orientan la investigación en (IS): Transferencia de conocimiento; La transferencia de conocimiento como factor positivo para la organización; Transferencia de conocimiento e innovación estratégica.

Aportes teóricos / metodológicos: se pudo identificar que no existía un artículo de revisión sobre el tema (IS). Entonces, este estudio pudo contribuir con la teoría y mostrar los trabajos existentes que tuvieron una mayor influencia en (IS).

Contribuciones sociales / para la gestión: se advierte que los artículos existentes abordan (IS) como la dificultad de la transferencia de conocimiento. Con esto, este estudio también puede contribuir a la práctica de la transferencia de conocimiento, principalmente porque este tema está relacionado con el desarrollo de la ventaja competitiva en las empresas.

Palabras clave: Internal Stickiness; Transferencia de conocimiento; Bibliométrica. 


\section{INTRODUÇÃO}

A transferência de conhecimento e melhores práticas é considerada fundamental para o funcionamento efetivo das organizações (Osterloh \& Frey, 2000; Tallman, Jenkins, Henry, \& Pinch, 2004). No entanto, a prática mostra que apesar do reconhecimento dessa importância, esta transferência é bastante difícil. A dificuldade de transferir o conhecimento e melhores práticas nas organizações é representada pelo termo Internal Stickiness - (IS) (Szulanski, 1996).

O conceito de Stickiness tem o significado de viscosidade. Na administração, é uma metáfora que vem da dificuldade para circular um fluido, para a dificuldade de transferir o conhecimento (Schuller, 2014). O pesquisador Szulanski (1996) estava interessado em compreender o que impedia a transferência de conhecimento e melhores práticas nas organizações. O autor defende que existem outros fatores, que somente a ausência de incentivo, que impedem a transferência, e que dependem de onde ou de quem vem, quem detém, e do contexto organizacional que qualquer transferência ocorre. Estes fatores contribuem para a (IS).

A importância do conceito é reconhecida pela academia, visto que o trabalho de Szulanski (1996) foi laureado com o prêmio de melhor artigo do Strategic Management Journal em 2004. Apesar disso, o conceito da (IS) tem sido muito citado, mas pouco explorado (Jensen \& Szulanski, 2004; Szulanski, 1996, 2000; 2003; Szulanski, Ringov, \& Jensen, 2016). Em uma busca sumária pelo termo na base Web of Science (WoS), aparecem somente dois artigos sobre o tema, porém mais 5.000 citações ao termo integral por outros autores. Em especial, embora o artigo de Szulanski (1996) tenha focado na transferência de práticas, o autor indica na literatura a transferência de conhecimento tendo como objeto as barreiras que ocorrem nos projetos dessas transferências.

O presente estudo teve como objetivo identificar a estrutura intelectual da pesquisa sobre (IS) a partir das referências existentes nas publicações sobre esse tema. Propõe-se a investigar os trabalhos que influenciam e influenciaram o conceito (Vogel \& Güttel, 2012), pela condução de um estudo bibliométrico sobre a (IS). Neste estudo, serão usadas as técnicas de citação e cocitação bibliográfica, apoiada por meio da análise fatorial e de redes.

Este artigo é dividido da seguinte forma: na primeira parte são apresentados a contextualização e uma breve explanação sobre o conceito da (IS). $\mathrm{Na}$ sequência descreve se a importância da transferência de conhecimento nas organizações e a aplicação de uma revisão de literatura sobre o tema estudado. $\mathrm{Na}$ seção seguinte são descritos os procedimentos metodológicos utilizados para esta pesquisa. $\mathrm{Na}$ sequência são apresentados as discussões e os resultados da análise das citações nos periódicos, a análise das cargas fatoriais e o mapa de rede com suas relações. Por último são apresentados itens que dão suporte as conclusões e contribuições sobre o conceito da (IS).

\section{REFERENCIAL TEÓRICO}

O conhecimento é um recurso organizacional relevante para o crescimento, produtividade e sobrevivência de uma organização (Szulanski et al., 2016). As organizações e suas filiais aprendem e trocam conhecimentos entre si, que geralmente são mais competitivas, produtivas e com maior índice de sobrevivência em relação as organizações não movidas à transferência de conhecimento (Argote, 2012). Vários autores se dedicaram a estudar a transferência de conhecimento. Talvez os autores mais clássicos tenham sido Nonaka e Takeushi (1995), que argumentam que a transferência de conhecimento é um processo social com uma interação contínua e dinâmica entre conhecimento tácito e explícito. Este processo passa a ser influenciado, por exemplo, por quão tácito é o conhecimento (Akbar, 2003) e por aspectos psicológicos (Andrews \& Delahaye, 2000), dentre outros.

Outro aspecto é o processo de inovação, dentro das organizações estão relacionados a transferência eficaz de conhecimento entre seus colaboradores (Jasimuddin, 2007). Isso, porque a competitividade das organizações está relacionada ao processo de inovação dentro de uma organização (Argote \& Ingram, 2000). Diante desse contexto, Szulanski (1996, 2000, 2003) propõe o conceito da (IS), como a dificuldade de transferência de conhecimento e melhores práticas, em função de barreiras à essa 
transferência. Os pesquisadores Das \& Chakraborty, (2018) descrevem o conceito de retenção de conhecimento como interrompimento de transferência, e conceitos ligados a ocultação de conhecimento e barreiras na transferência de conhecimento. O processo de transferência de conhecimento não é automático. Embora, Szulanski (1996) aborda que a motivação possa afetar a transferência de conhecimento, o autor atribui à ambiguidade causal e às incertezas de comunicação como fatores preponderantes.

No entanto, afirmam Wang \& Wang, (2012) que o processo de inovação de uma organização depende dos seus membros com seus conhecimentos, habilidades e experiências, ou seja, a transferência de conhecimento gera valor para a organização. Szulanski (1996) apresenta um processo de quatro estágios de transferência de tecnologia: iniciação, implementação, construção e integração. O processo de transferência se inicia quando há o conhecimento, e existem a necessidade para a utilização deste conhecimento. O surgimento desta necessidade pode tornar nítido possíveis soluções, na busca do aparecimento de um amplo conhecimento. Na utilização da ferramenta benchmarking, a comparação dos resultados desses amplos conhecimentos revelará qual é o melhor (Balm, 1992). Essa revelação pode iniciar uma melhor averiguação dos resultados e uma possível solução na comprovação dessa necessidade, sendo explorada a acessibilidade da transferência (Szulanski, 1996).

Havendo a necessidade de usar e agir em relação ao uso, se inicia a implementação. Na implementação os recursos fluem entre o receptor e a fonte do conhecimento. Os relacionamentos específicos da transferência entre o receptor e a fonte são determinados, transferidos e ajustados conforme as necessidades do receptor, usando como exemplo problemas já constatados de outra transferência da mesma prática, ou auxiliando no início de novo conhecimento com menor hostilidade ao receptor (Buttolph, 1992; Rice \& Rogers, 1980). Szulanski, (1996) afirma que ao receptor começar a fazer uso do conhecimento transferido, automaticamente, a operação relacionada à implementação finalizam ou será reduzida.

O início da construção acontece quando o receptor começa a utilizar o conhecimento, aprender com os problemas, para ir melhorando o desempenho e conseguir os resultados esperados.
No início, é quase certo que o receptor utilize este novo conhecimento de forma não eficaz (Adler, 1990; Baloff, 1970; Chew, 1991; Galbraith, 1990; LeonardBarton, Chew \& Bohn, 1991), porém, aos poucos melhorando o desempenho, chegando ao um nível aceitável. O início da construção é uma fase que permite por um breve período, uma solução para problemas que poderão surgir (Tyre \& Orlikowski, 1994).

A integração se inicia com os resultados satisfatórios, o que indica a institucionalização do conhecimento. A utilização do conhecimento adquirido progressivamente é o início dos processos sociais (Berger \& Luckman, 1966). Descreve Szulanski, (1996) que com o tempo o compartilhamento do conhecimento é construído no receptor, sendo associados ações e atores. Esses compartilhamentos auxiliam no gerenciamento dos processos, de forma prática, esperável e aceitável (March \& Simon, 1958; Nelson \& Winter, 1982; Tolbert, 1987). Cada um dos estágios é socialmente interligado com ações, interações e práticas relacionadas aos seres humanos.

No entanto, existem barreiras à transferência de conhecimento. Szulanski (1996) identifica barreiras ligadas às características do conhecimento transferido: ignorância; capacidade absortiva; falta de relacionamento pré-existente; falta de motivação. A ignorância indica que aqueles que detêm o conhecimento não se dão conta que o conhecimento possa ser considerado útil por outros, e aqueles que poderiam se beneficiar do conhecimento não tem ideia que ele esteja disponível. Pode não haver capacidade absortiva, por falta de recursos financeiros, recursos gerenciais e tempo disponível para tornar o conhecimento utilizável.

Os relacionamentos pré-existente entre as pessoas é que viabiliza a transferência de conhecimento. Se não acontecer, o conhecimento não será transferido. A falta de motivação acontece pelo fato de não haver a percepção de razões de negócio para que aconteça a transferência de conhecimento e de melhores práticas. Uma das particularidades da fonte de conhecimento pode se opor em transferir conhecimento, talvez pensando que irá ser prejudicado em perder seu cargo, não ser premiado por transferir esse conhecimento ou não dispor de tempo para compartilhar esse conhecimento (Szulanski, 1996). 
Uma das particularidades do receptor do conhecimento é a ausência da capacidade absortiva, não conseguindo absorver das fontes externas do conhecimento (Cohen \& Levinthal, 1990). Entretanto, está capacidade funciona como uma reserva de armazenamento de conhecimento, na qual, valoriza o entendimento e pratica os novos conhecimentos nos negócios (Dierickx \& Cool, 1989). Outra particularidade de alguns receptores do conhecimento é a ausência de motivação em acatar o conhecimento exterior (Hayes \& Clark, 1985). A ausência de motivação pode contribuir para uma aceitação falsa, desonesta e recusa na implementação de novos conhecimentos (Zaltman, Duncan \& Holbek, 1973).

\section{MÉTODO}

O método deste trabalho tem como base o que foi proposto por Serra, Ferreira, Guerrazzi \& Scaciotta (2018). Foi utilizada como método a bibliometria, pois ele permite a união de vários dados bibliográficos por meio de análises estatísticas (Vogel \& Güttel, 2012; Zupic \& Čater, 2015). Neste estudo, serão usadas as técnicas de citação e cocitação bibliográfica, apoiada por meio da análise fatorial e de redes.

\section{Procedimentos de coleta de dados}

Os dados dos artigos selecionados para este trabalho foram coletados na base da ISI Web of Science entre o ano de 1994 (quando as pesquisas sobre stickiness iniciaram) e até 2018 (ano de estudo deste artigo). Para a seleção, foi utilizada a palavra chave "stickiness" no campo topic. O campo topic delimita a busca entre título, resumo e palavraschave dos artigos. Com essa seleção apareceram a palavra "stickiness" em mais de 3800 publicações.

Após esta etapa, foi necessário um ajuste no comando de seleção do WoS para eliminar, o máximo possível, os artigos que provavelmente não fossem do tema. Foram selecionados todos os periódicos de business, management e social sciences interdisciplinary disponíveis no Journal Citation Reports da base. A amostra foi de 236 artigos que apareceram a palavra "stickiness". Depois dessas seleções, foi importante uma leitura dos resumos para verificar se realmente os artigos eram sobre a área do tema estudado. Com isso, foram eliminados 84 artigos e restando a amostra final de 152 artigos sobre o tema estudado.

Na Figura 1 é possível visualizar a evolução das publicações dos artigos sobre o tema (IS) durando o período de 1994 a 2018. Nota-se uma quantidade significativa de artigos nos últimos vinte quatro anos, e crescente a partir da década de 2000. Entretanto, houve uma queda em publicações no ano 2005 e no ano de 2001 ficando sem registros de publicações. Mas, o tema (IS) conquistou seu maior patamar nos anos de 2017 e 2018.

Figura 1 Evolução das publicações na base da ISI Web of Science.

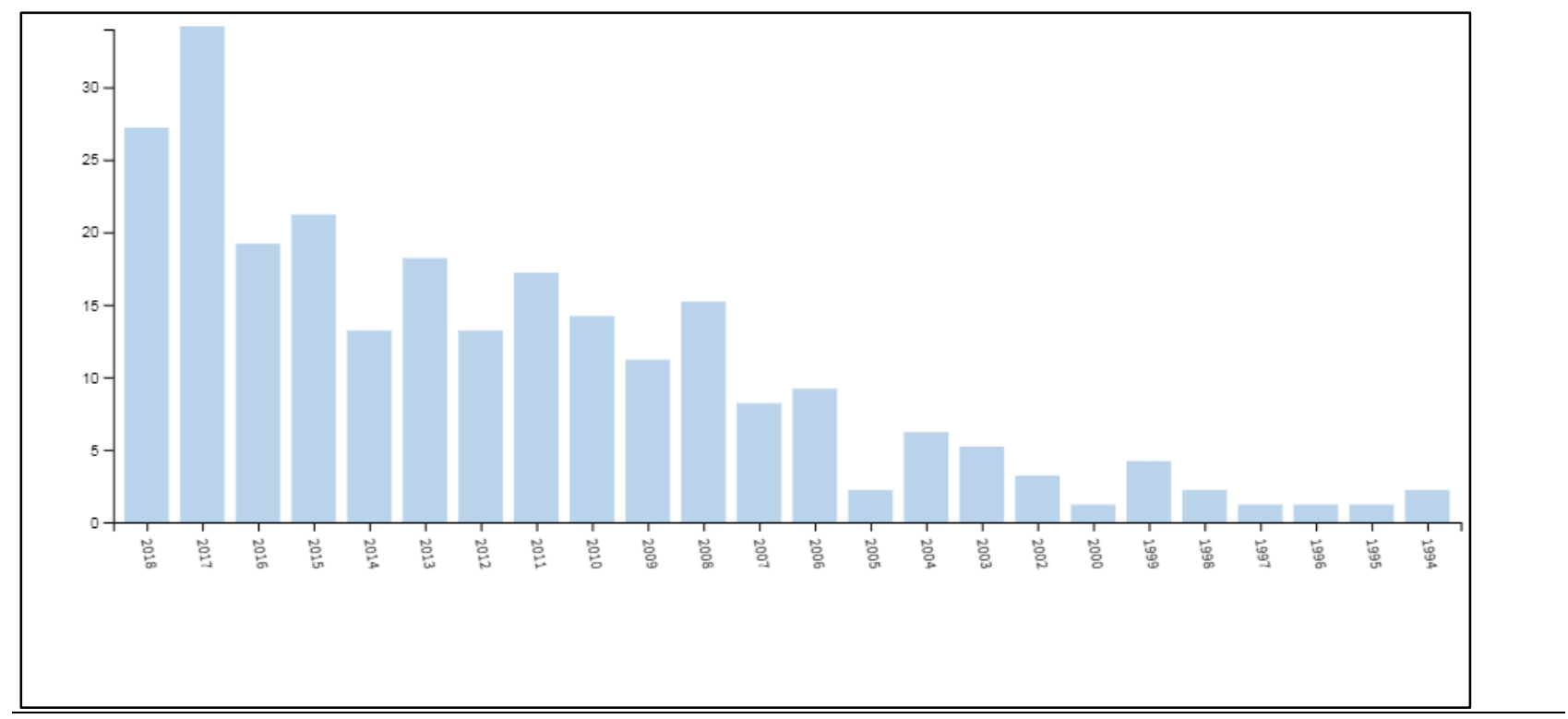

Intern. Journal of Profess. Bus. Review; São Paulo V.6 N.1 2021, pp. 1-19, Jan/Dec.2021 
Fonte: Base da ISI Web of Science.

\section{Procedimentos de análise}

Primeiramente, foi realizada uma análise de citação com o uso do software Bibexcel (Pilkington \& Meredith, 2009), na qual foram identificados os trabalhos mais representativos no campo por serem estes os mais citados. A análise de citações é a frequência com que os trabalhos individuais são referenciados em demais trabalhos (Vogel \& Güttel, 2012).

A análise de cocitação apresenta a semelhança a partir da frequência em que dois trabalhos são referenciados em conjunto por outros trabalhos (McCain, 1990; Small, 1973). Na análise de cocitação ao longo do tempo pode sofrer alteração, com isto, contribuindo para verificar as alterações de paradigmas e abordagens teóricas de determinado tema (Zupic \& Čater, 2015).

Na etapa de análise da citação, foi trabalhada a matriz que serviu de base para a análise de cocitação. Dessa forma, esta matriz teve que ser trabalhada e preparada com o agrupamento de referências que possam ser a mesma, mas, escritas de maneira diferentes. Além deste tratamento, o arquivo sofreu um corte de referências dos trabalhos mais citados na ordem de $6 \%$ a $10 \%$, conforme lei de bibliometria de Lotka (Urbizagástegui Alvarado, 2002) significa que mais citados dos autores dos artigos no tema teriam representatividade no campo estudado. Com isso, obteve-se os 44 trabalhos mais citados que representam $13 \%$ do total, sendo maior do que o estabelecido (Nath \& Jackson, 1991), constituindo um corte de até 10 citações.

Assim, para a análise de cocitação foi usada essa matriz gerada no software Bibexcel e que teve este primeiro tratamento, mencionado anteriormente. Para gerar a cocitação por meio da análise fatorial no software SPSS e o mapa de rede nos softwares Ucinet e Pajek, a matriz foi ajustada novamente, seguindo os padrões de referências da American Psychological Association, 6th edition (APA). A análise fatorial tem sido utilizada para dar coerência e vigor aos trabalhos bibliométricos (McCain, 1990). Nesta verificação foi utilizada análise fatorial com rotação Varimax (Acedo, Barroso, \& Galan, 2006; Lin \& Cheng, 2010), a qual assegura melhores acepções (Fabrigar, Wegener, MacCallum, \& Strahan, 1999). Também foram apreciadas cargas fatoriais superiores ou iguais a 0,4 (Guerrazzi, Brandão, Junior, \& Lourenço, 2015; Shafique, 2013) para a escolha dos fatores que compõe a análise de cocitação.

Os artigos que compõem cada fator foram lidos para que, a partir da compreensão do conjunto do conteúdo, pudessem ser nomeados. Os fatores foram utilizados e aplicados na representação de redes pelos softwares Ucinet e Pajek, outro método muito utilizado em estudos bibliométricos (Lin \& Cheng, 2010; Ramos-Rodríguez \& Ruíz-Navarro, 2004). A rede mostra a continuidade entre artigos e as suas conexões, o que representa a ligação e a proporção da quantidade de citações em conjunto. A análise fatorial foi realizada com o uso do software SPSS versão 2.0 e a rede de cocitação com o uso dos softwares Ucinet e Pajek. Com isso, gerou-se uma matriz de co-ocorrências das referências da amostra e foi possível verificar a influência das raízes intelectuais (cocitação) e representar as relações graficamente.

\section{RESULTADOS E DISCUSSÕES}

Nesta seção serão apresentadas as análises (citação / cocitação) e discussões dos resultados. $\mathrm{Na}$ Tabela 1, os resultados indicam uma predominância de publicação dos artigos citados sobre o tema estudado em periódicos com foco em Pesquisa e Prática em Gestão do Conhecimento, Pesquisas Empresariais, Estudos Internacionais de Negócios e Gestão Estratégica. 
Tabela 1 Periódicos e quantidade de artigos selecionados.

\begin{tabular}{|c|c|c|}
\hline Periódicos & $\begin{array}{c}\text { Total } \\
\text { Artigos } \\
\text { Amostra }\end{array}$ & $\%$ Amostra \\
\hline Knowledge Management Research \& Practice & 8 & $5 \%$ \\
\hline Journal of International Business Studies & 5 & $3 \%$ \\
\hline Strategic Management Journal & 5 & $3 \%$ \\
\hline Research Policy & 4 & $3 \%$ \\
\hline International Business Review & 3 & $2 \%$ \\
\hline International Journal of Human Resource Management & 3 & $2 \%$ \\
\hline International Journal of Operations \& Production Management & 3 & $2 \%$ \\
\hline International Journal of Technology Management & 3 & $2 \%$ \\
\hline Journal of Knowledge Management & 3 & $2 \%$ \\
\hline Management Learning & 3 & $2 \%$ \\
\hline Management Science & 3 & $2 \%$ \\
\hline Organization Science & 3 & $2 \%$ \\
\hline $\begin{array}{l}\text { Canadian Journal of Administrative Sciences-Revue Canadienne des Sciences } \\
\text { de } L \text { Administration }\end{array}$ & 2 & $1 \%$ \\
\hline Industrial and Corporate Change & 2 & $1 \%$ \\
\hline Information \& Management & 2 & $1 \%$ \\
\hline International Journal of Management Reviews & 2 & $1 \%$ \\
\hline Journal of International Management & 2 & $1 \%$ \\
\hline Journal of Organizational Change Management & 2 & $1 \%$ \\
\hline Journal of Strategic Information Systems & 2 & $1 \%$ \\
\hline Management Decision & 2 & $1 \%$ \\
\hline Management International Review & 2 & $1 \%$ \\
\hline Other Newspapers, Books, Conferences and Symposium & 31 & $20 \%$ \\
\hline TOTAL & 152 & $100 \%$ \\
\hline
\end{tabular}

Fonte: Elaborado pelos autores.

Identificou-se, que os periódicos com a maior concentração dos estudos sobre (IS) são: o Knowledge Management Research \& Practice com oito artigos; Journal of International Business Studies e Strategic Management Journal, ambos com cinco artigos; Research Policy com quatro artigos; e outros periódicos nas áreas Human Resource Management, Operations \& Production Management, Technology Management, Management Learning, e etc.

$\mathrm{Na}$ Tabela 2, pode-se verificar os 44 artigos mais citados, que possuem a partir de dez citações e trabalhos que foram objeto da análise. Observe que os trabalhos de (Szulanski, 1996, 2000) foram os trabalhos mais citados sobre o conceito da (IS). Outros trabalhos relevantes foram citados confirmando as barreiras na transferência de conhecimento (Argote \& Ingram, 2000; Cohen \& Levinthal, 1990; Jensen \& Szulanski, 2004). Os autores (Gupta \& Govindarajan, 2000) abordam sobre as barreiras adicionais à transferência de conhecimento e a relevância deste compartilhamento de conhecimento. 
Tabela 2 Frequência de citação dos artigos utilizados nas referências.

\begin{tabular}{|c|c|c|}
\hline Referências completas & Amt. & $\begin{array}{l}\% \text { nos } 152 \\
\text { artigos }\end{array}$ \\
\hline $\begin{array}{l}\text { Szulanski, G. (1996). Exploring internal stickiness: Impedi-ments to the transfer of } \\
\text { best practice within the firm. Strategic Management Journal, 17: 27-44. }\end{array}$ & 108 & $71,05 \%$ \\
\hline $\begin{array}{l}\text { Szulanski, G. (2000). The process of knowledge transfer: a diachronic analysis of } \\
\text { stickiness. Organizational Behavior and Human Decision Processes 82: 9-27. }\end{array}$ & 83 & $54,61 \%$ \\
\hline $\begin{array}{l}\text { Cohen, W. M., \& Levinthal, D. A. (1990). Absorptive Capacity: A New Perspective on } \\
\text { Learning and Innovation. Administrative Science Quarterly, } 35 \text { (1), 128-152. }\end{array}$ & 57 & $37,50 \%$ \\
\hline $\begin{array}{l}\text { Nonaka, I. (1994). A dynamic theory of organizational knowledge creation. } \\
\text { Organization Science, 5, pp. 14-37. }\end{array}$ & 41 & $26,97 \%$ \\
\hline $\begin{array}{l}\text { Argote, L., \& Ingram, P. (2000). Knowledge transfer: A basis for competitive } \\
\text { advantage in firms. Organizational Behavior and Human Decision Processes. 82(1), } \\
\text { 150-169. }\end{array}$ & 36 & $23,68 \%$ \\
\hline $\begin{array}{l}\text { Nonaka, I., Takeuchi, H. (1995). The Knowledge-Creating Company. Oxford University } \\
\text { Press, New York and Oxford, UK. }\end{array}$ & 36 & $23,68 \%$ \\
\hline $\begin{array}{l}\text { Jensen, R. \& Szulanski, G. (2004). Stickiness and the adaptation of organizational } \\
\text { practices in cross-border knowledge transfers. Journal of International Business } \\
\text { Studies. } 35(6), 508-523 .\end{array}$ & 31 & $20,39 \%$ \\
\hline $\begin{array}{l}\text { Von Hippel, E. (1994). "Sticky Information" and the Locus of Problem Solving: } \\
\text { Implications for Innovation. Management Science. 40(4):429-439. }\end{array}$ & 31 & $20,39 \%$ \\
\hline $\begin{array}{l}\text { Gupta, A. K. \& Govindarajan, V. (2000). Knowledge flows within multinational } \\
\text { corporations. Strategic Management Journal, } 21 \text { (4), 473-496. }\end{array}$ & 28 & $18,42 \%$ \\
\hline $\begin{array}{l}\text { Nelson, R., \& Winter, S. (1982). An evolutionary theory of economic change. } \\
\text { Cambridge: Belknap Press. }\end{array}$ & 28 & $18,42 \%$ \\
\hline $\begin{array}{l}\text { Kogut, B., \& Zander, U. (1992). Knowledge of the firm, combinative capabilities and } \\
\text { the replication of technology. Organization Science, 3(3), 383-397. }\end{array}$ & 27 & $17,76 \%$ \\
\hline $\begin{array}{l}\text { Zander, U., \& Kogut, B. (1995). Knowledge and the speed of the transfer and imitation } \\
\text { of organizational capabilities: An empirical test. Organization Science, 6(1) 76-92. }\end{array}$ & 27 & $17,76 \%$ \\
\hline $\begin{array}{l}\text { Simonin, B. L. (1999). Ambiguity and the Process of Knowledge Transfer in Strategic } \\
\text { Alliances. Strategic Management Journal. 20: 595-623. }\end{array}$ & 26 & $17,11 \%$ \\
\hline $\begin{array}{l}\text { Grant, R. M. (1996). Toward a knowledge-based theory of the firm. Strategic } \\
\text { Management J. } 17 \text { 109-122. }\end{array}$ & 25 & $16,45 \%$ \\
\hline $\begin{array}{l}\text { Hansen, M. (1999). The search-transfer problem: The role of weak ties in sharing } \\
\text { knowledge across organization subunits. Administrative Science Quarterly, 44(1) 82- } \\
111 .\end{array}$ & 25 & $16,45 \%$ \\
\hline $\begin{array}{l}\text { Davenport, T. H. \& Prusak, L. (1998). Working knowledge: how organizations manage } \\
\text { what they know. Harvard Business School Press. Boston, Massachusetts. }\end{array}$ & 18 & $11,84 \%$ \\
\hline $\begin{array}{l}\text { Minbaeva, D., Pedersen, T., Björkman, I, Fey, C. F., \& Park, H. J. (2003). MNC } \\
\text { knowledge transfer, subsidiary absorptive capacity and HRM. Journal of International } \\
\text { Business Studies, 34, 586-59. }\end{array}$ & 18 & $11,84 \%$ \\
\hline $\begin{array}{l}\text { Kogut, B \& Zander, U. (1993). Knowledge of the firm and the evolutionary theory of } \\
\text { the multinational corporation. Journal of International Business Studies, } 24 \text { (4), 625- } \\
645 .\end{array}$ & 17 & $11,18 \%$ \\
\hline $\begin{array}{l}\text { Kostova, T. (1999). Transnational transfer of strategic organizational practices: A } \\
\text { contextual perspective. Academy of Management Review, 24(2), 308-324. }\end{array}$ & 16 & $10,53 \%$ \\
\hline $\begin{array}{l}\text { Kostova, T. \& Roth, K. (2002). Adoption of an Organizational Practice by Subsidiaries } \\
\text { of Multinational Corporations: Institutional and Relational Effects. The Academy of } \\
\text { Management Journal. } 45 \text { (1), 215-233. }\end{array}$ & 16 & $10,53 \%$ \\
\hline
\end{tabular}




\begin{tabular}{|c|c|c|}
\hline $\begin{array}{l}\text { Lippman, S. A., \& Rumelt, R. P. (1982). Uncertain Imitability: an analysis of interfirm } \\
\text { differences in efficiency under competition. Bell Journal of Economics, 13, 418-438. }\end{array}$ & 16 & $10,53 \%$ \\
\hline $\begin{array}{l}\text { Cummings, J. L., \& Teng, B. S. (2003). Transferring R\&D knowledge: the key factor } \\
\text { affecting knowledge transfer success. Journal of Engineering and Technology } \\
\text { Management, } 20(1-2) \text {, 39-68. }\end{array}$ & 14 & $9,21 \%$ \\
\hline $\begin{array}{l}\text { Eisenhardt, K. M. (1989). Building Theories from Case Study Research. The Academy } \\
\text { of Management Review, } 14 \text { (4), 532-550. }\end{array}$ & 14 & $9,21 \%$ \\
\hline $\begin{array}{l}\text { Mowery, D. C., Oxley, J. E., \& Silverman, B. S. (1996). Strategic alliances and interfirm } \\
\text { knowledge transfer. Strategic Management Journal, Vol. 17(Winter Special Issue), } \\
\text { 77-91. }\end{array}$ & 13 & $8,55 \%$ \\
\hline Polanyi, M. (1966). The Tacit Dimension. Routledge and Kegan Paul, London, UK. & 13 & $8,55 \%$ \\
\hline $\begin{array}{l}\text { Teece, D. J., Pisano, G., \& Shuen, A. (1997). Dynamic capabilities and strategic } \\
\text { management. Strategic Management Journal, } 18 \text { (7), 509-533. }\end{array}$ & 13 & $8,55 \%$ \\
\hline $\begin{array}{l}\text { Osterloh, M. \& Frey, B.S. (2000). Motivation, Knowledge Transfer, and Organizational } \\
\text { Forms. Organization Science, } 11 \text { (5), 538-550. }\end{array}$ & 12 & $7,89 \%$ \\
\hline $\begin{array}{l}\text { Tsai, W. (2001). Knowledge transfer in intraorganizational networks: Effects of } \\
\text { network position and absorptive capacity on business unit innovation and } \\
\text { performance. The Academy of Management Journal, } 44(5), 996-1004 \text {. }\end{array}$ & 12 & $7,89 \%$ \\
\hline $\begin{array}{l}\text { Winter, S.G., \& Szulanski, G. (2001). Replication as Strategy, Organization Science. } \\
12(6) 730-743 .\end{array}$ & 12 & $7,89 \%$ \\
\hline $\begin{array}{l}\text { Zahra, S. A., \& George, G. (2002). Absorptive capacity: a review, reconceptualization, } \\
\text { and extension. Academy of Management Review. } 27 \text { (2), 185-203. }\end{array}$ & 12 & $7,89 \%$ \\
\hline $\begin{array}{l}\text { Barney, J., (1991). Firm Resources and Sustained Competitive Advantage. Journal of } \\
\text { Management. 17(1), 99-120. }\end{array}$ & 11 & $7,24 \%$ \\
\hline $\begin{array}{l}\text { Bartlett, C. A., \& Ghoshal, S. (1989). Managing Across Borders: The Transnational } \\
\text { Solution. Harvard Business School Press. }\end{array}$ & 11 & $7,24 \%$ \\
\hline March, J., \& Simon, H. (1958). Organizations. New York: Wiley. & 11 & $7,24 \%$ \\
\hline $\begin{array}{l}\text { Reed, R. \& DeFillippi, R. J. (1990). Causal Ambiguity, Barriers to Imitation, and } \\
\text { Sustainable Competitive Advantage. The Academy of Management Review. 15(1), 88- } \\
\text { 102. }\end{array}$ & 11 & $7,24 \%$ \\
\hline $\begin{array}{l}\text { Teece, D. J. (1977). Technology transfer by multinational firms: The resource cost of } \\
\text { transferring technological know-how. The Economic Journal, } 87(346), 242-261 .\end{array}$ & 11 & $7,24 \%$ \\
\hline $\begin{array}{l}\text { Argote L, McEvily B, Reagans R (2003) Managing knowledge in organizations: An } \\
\text { integrative framework and review of emerging themes. Management Sci. 49(4):571- } \\
582 \text {. }\end{array}$ & 10 & $6,58 \%$ \\
\hline $\begin{array}{l}\text { Bresman, H., Birkinshaw, J., \& Nobel, R. (1999). Knowledge Transfer In International } \\
\text { Acquisitions. Journal of International Business Studies, } 30 \text { (3), 439-462. }\end{array}$ & 10 & $6,58 \%$ \\
\hline $\begin{array}{l}\text { Galbraith, C.S., (1990). Transferring core manufacturing technologies in high tech } \\
\text { firms. California Management Review } 32(4), 56-70 .\end{array}$ & 10 & $6,58 \%$ \\
\hline $\begin{array}{l}\text { Goh, S. C. (2002). Managing effective Knowledge transfer: an integrative framework } \\
\text { and some practice implications. Journal of Knowledge Management. } 6 \text { (1), 23-30. }\end{array}$ & 10 & $6,58 \%$ \\
\hline $\begin{array}{l}\text { Lane, P. J. \& Lubatkin, M. (1998). Relative absorptive capacity and interorganizational } \\
\text { learning. Strategic Management Journal. 19:461-477. }\end{array}$ & 10 & $6,58 \%$ \\
\hline $\begin{array}{l}\text { March, J. G. (1991). Exploration and exploitation in organizational learning. Organ. } \\
\text { Sci. 2(1) 71-87. }\end{array}$ & 10 & $6,58 \%$ \\
\hline $\begin{array}{l}\text { Reagans, R. \& McEvily, B. (2003). Network Structure and Knowledge Transfer: The } \\
\text { Effects of Cohesion and Range. Administrative Science Quarterly. 48: } 240 .\end{array}$ & 10 & $6,58 \%$ \\
\hline Szulanski, G. (2003). Sticky knowledge: Barriers to knowing in the firm. London: Sage. & 10 & $6,58 \%$ \\
\hline $\begin{array}{l}\text { Szulanski, G., Cappetta, R., \& Jensen, R. J. (2004). When and How Trustworthiness } \\
\text { Matters: Knowledge Transfer and the Moderating Effect of Causal Ambiguity. } \\
\text { Organization Science, } 15 \text { (5), 600-613. }\end{array}$ & 10 & $6,58 \%$ \\
\hline
\end{tabular}

Fonte: Elaborado pelos autores. 
The integrity of the upright guides them. (Proverbs 11:3)

Executive Editor: Altieres de Oliveira Silva

Associate Editor: Manuel Portugal Ferreira, Ph.D.

Evaluation Process: Double Blind Review pelo SEER/OJS

A matriz de componente rotativa nos permitiu a identificação de 3 fatores responsáveis por 77,69\% de variância. O resultado da análise de componentes é apresentado na Tabela 3. O resultado da análise de componente rotativa considera referências agrupadas nos 3 fatores conforme carga fatorial $(>=0,4)$, prevalecendo a maior carga conforme cada fator. O primeiro, fator 1 é o predominante com 22 artigos, pois concentra a maioria das referências. $O$ fator 2 apresentam-se referências em 9 artigos. $O$ fator 3 coincide com o fator anterior apresentando referências em outros 9 artigos. A seguir, pode-se visualizar a Tabela 3 com o resultado da análise fatorial de cocitação.

Tabela 3 Resultados da Análise Fatorial de Cocitação.

\begin{tabular}{|l|l|l|l|}
\hline Matriz de componente rotativaa & \multicolumn{2}{l}{ Componente } \\
\cline { 2 - 4 } & Fator \#1 & Fator \#2 & Fator \#3 \\
\hline Osterloh \& Frey (2000) & 0,866 & 0,203 & 0,162 \\
\hline Cummings \& Teng (2003) & 0,862 & 0,237 & 0,216 \\
\hline Reagans \& McEvily (2003) & 0,846 & 0,257 & 0,177 \\
\hline Goh (2002) & 0,829 & 0,305 & 0,156 \\
\hline Davenport \& Prusak (1998) & 0,804 & 0,431 & 0,148 \\
\hline Bresman \& Birkinshaw \& Nobel (1999) & 0,772 & 0,298 & 0,379 \\
\hline Zahra \& George (2002) & 0,752 & 0,159 & 0,468 \\
\hline Polanyi (1966) & 0,749 & 0,237 & 0,466 \\
\hline Argote \& Ingram (2000) & 0,733 & 0,470 & 0,337 \\
\hline Tsai (2001) & 0,728 & 0,450 & 0,249 \\
\hline Nonaka \& Takeuchi (1995) & 0,697 & 0,522 & 0,353 \\
\hline Mowery \& Oxley \& Silverman (1996) & 0,655 & 0,262 & 0,584 \\
\hline Nonaka (1994) & 0,637 & 0,462 & 0,421 \\
\hline Grant (1996) & 0,636 & 0,567 & 0,358 \\
\hline Hansen (1999) & 0,628 & 0,542 & 0,373 \\
\hline Kogut \& Zander (1992) & 0,586 & 0,516 & 0,477 \\
\hline Minbaeva \& Pedersen \& Bjorkman \& Fey \& Park (2003) & 0,584 & 0,556 & 0,395 \\
\hline Vonhippel (1994) & 0,584 & 0,435 & 0,506 \\
\hline Zander \& Kogut (1995) & 0,545 & 0,458 & 0,526 \\
\hline Szulanski (2000) & 0,519 & 0,508 & 0,270 \\
\hline Szulanski (2003) & 0,518 & 0,445 & 0,448 \\
\hline Szulanski (1996) & 0,455 & 0,120 & 0,426 \\
\hline Kostova \& Roth (2002) & 0,322 & 0,755 & 0,289 \\
\hline Kogut \& Zander (1993) & 0,351 & 0,746 & 0,342 \\
\hline Eisenhardt (1989) & 0,402 & 0,730 & 0,336 \\
\hline Bartlett \& Ghoshal (1989) & 0,038 & 0,729 & 0,479 \\
\hline Kostova (1999) & 0,355 & 0,700 & 0,391 \\
\hline Argote \& McEvily \& Reagans (2003) & 0,504 & 0,675 & 0,262 \\
\hline Jensen \& Szulanski (2004) & 0,493 & 0,650 & 0,368 \\
\hline & & & \\
\hline
\end{tabular}




\begin{tabular}{|l|l|l|l|}
\hline Barney (1991) & 0,401 & 0,596 & 0,516 \\
\hline Cohen \& Levinthal (1990) & 0,552 & 0,590 & 0,289 \\
\hline March \& Simon (1958) & 0,008 & 0,314 & 0,789 \\
\hline Galbraith (1990) & 0,379 & 0,241 & 0,780 \\
\hline Lippman \& Rumelt (1982) & 0,272 & 0,343 & 0,767 \\
\hline Reed \& Defillippi (1990) & 0,301 & 0,298 & 0,760 \\
\hline Teece (1977) & 0,389 & 0,263 & 0,748 \\
\hline Winter \& Szulanski (2001) & 0,236 & 0,480 & 0,637 \\
\hline Szulanski \& Cappetta \& Jensen (2004) & 0,364 & 0,440 & 0,637 \\
\hline March (1991) & 0,304 & 0,516 & 0,609 \\
\hline Nelson \& Winter (1982) & 0,416 & 0,491 & 0,560 \\
\hline
\end{tabular}

Fonte: Elaborado pelos autores - software SPSS versão 2.0.

Com o intuito de compreender a análise fatorial, todos os 40 artigos foram lidos e interpretados. Dessa forma, foi possível obter uma visão geral e que proporcionou o entendimento da base teórica, e as discussões que os autores realizaram sobre o tema (IS). Assim, observe alguns estudos realizados pelos autores citados nos três fatores resultados da análise fatorial de cocitação.

Após a leitura dos 40 artigos, que resultaram da análise de cocitação, e foram agrupados após tratamento da análise fatorial, é possível perceber que o fator 1 é o predominante com 22 artigos. Este fator foi denominado de "Transferência de Conhecimento". Neste fator, nitidamente, os autores estudaram a transferência de conhecimento como tentativa de entendê-la melhor (Cummings \& Teng, 2003; Goh, 2002; Osterloh \& Frey, 2000). Outros procuraram explicações para transmitir ideias complexas, explicações para transferência de conhecimento com base na capacidade de absorção (Reagans \& McEvily, 2003). Alguns escreveram um verdadeiro manual para a gestão do conhecimento (Davenport \& Prusak, 1998). A transferência de conhecimento está também presente em aquisições internacionais (Bresman \& Birkinshaw \& Nobel, 1999). No entanto, a compreensão está relacionada aos processos, que permitem analisar as informações obtidas de fontes externas. E, que o conhecimento externo pode até impedir que pessoas de fora entendam ou compartilhe esse conhecimento (Zahra \& George, 2002).

O conhecimento sendo tácito é como algo que sabemos muito e dizemos pouco (Polanyi, 1966), ele é predominante na experiência e ideias de um indivíduo (Nonaka \& Takeuchi, 1995). Existem uma estrutura de reservatórios de conhecimento informando os tipos mais difíceis de processos de transferência para diversos contextos (Argote \& Ingram, 2000). Inclusive, até as organizações podem inovar e melhorar seu desempenho, ao centralizar na rede acesso a novos conhecimentos desenvolvidos por outras unidades das organizações (Tsai, 2001). Resultados revelam que laços fracos de interunidades, colaboram para que uma equipe de projeto busque conhecimento necessário em outras subunidades, mas que bloqueiam a transferência de conhecimento complexo, sendo necessário uma forte ligação entre as duas partes para uma transferência (Hansen, 1999). Alguns princípios de conhecimento recebido produz maior transferência, e a capacidade de absorção contribuem a detalhar a extensão de transferência de capacidade tecnológica (Mowery, Oxley, \& Silverman, 1996). Szulanski (1996) também aborda fortemente sobre a capacidade de transferir as boas práticas interna em uma empresa. Os resultados revelam barreiras à transferência interna de conhecimento como: ausência de capacidade de absorção do receptor, ambiguidade causal e uma relação intensa entre a fonte e o receptor.

Já o fator 2, denominado "Transferência de Conhecimento como fator positivo para a organização", com 9 artigos na análise fatorial. Os autores discutem sobre como a transferência de conhecimento pode influenciar positivamente no desempenho das organizações. Nota-se neste fator, alguns estudos empíricos (Jensen \& Szulanski, 2004) tentando testar esta relação. Outro assunto bem discutido neste fator é o de entender a transferência de conhecimento para a inovação (Bartlett \& Goshal, 1989), e com isso, gerar vantagem competitiva. A escolha do modo de transferência é determinada 
pela eficiência da organização multinacional na transferência de conhecimento em relação a outras empresas (Kogut \& Zander, 1993). A autora (Eisenhardt, 1989) é bastante citada nos trabalhos sobre (IS) devido seu artigo sobre o processo de indução de teoria usando estudos de caso. O método é utilizado para gerar e testar teoria, o mesmo tem ganhado força nas áreas de gestão e estratégia nas organizações.

Diante desses estudos, é recomendável que uma organização deve avaliar seus próprios ativos como: competitividade, flexibilidade e capacidade de aprendizagem em nível global (Bartlett \& Ghoshal, 1989). A vantagem competitiva surge de a impossibilidade de concorrentes implementarem estratégias de criação de valor (Barney, 1991). E, que a capacidade de uma organização adquirir conhecimento é fundamental para suas capacidades inovadoras (Cohen \& Levinthal, 1990). Argumentam os elementos da gestão do conhecimento e como representam a capacidade de uma unidade de criar, reter e transferir conhecimento (Argote, McEvily, \& Reagans, 2003).

E o fator 3, denominado "Transferência de Conhecimento e Inovação Estratégica", tem o mesmo número de artigos do fator anterior, sendo 9. Os estudos levam para a preocupação em entender a relação do conhecimento, como sendo um recurso essencial para a empresa, com a Ambiguidade Causal (Lippman \& Rumelt, 1982). A ambiguidade causal é vista como uma vantagem competitiva da empresa, pois dificulta como as concorrentes possam aprender (copiar) suas tecnologias. Os autores deste fator percebem que existe a necessidade de compreender melhor esses benefícios da transferência de conhecimento (Szulanski, Cappetta, \& Jensen, 2004), e como podem gerar vantagem para as empresas. Baseia-se em pesquisas empíricas da indústria (March \& Simon, 1958). Examinam o impacto de vários elementos sobre a economia, e consideram essas transferências de natureza intensa, e de alta tecnologia e inovação (Galbraith, 1990).

As empresas mais lucrativas podem estar asseguradas de novos entrantes e cujo êxito é difícil de ser imitado (Lippman \& Rumelt, 1982), levaram a um melhor entendimento da sustentabilidade das vantagens competitivas (Reed \& Defillippi, 1990). Verificou-se sobre o nível e os principais elementos da tecnologia, os custos relacionados na transferência de tecnologia e inovação (Teece, 1977). Examinou a correlação de possibilidades e a certezas na aprendizagem organizacional (March, 1991). Analisaram que a melhor utilização do conhecimento interno pode colaborar com a sobrevivência das empresas (Szulanski \& Cappetta \& Jensen, 2004). Propõem que uma teoria evolucionária é útil na análise de fenômenos associados as mudanças econômicas, mudanças na demanda de produtos ou condições de fornecimento de fatores, ou de inovação das empresas (Nelson \& Winter, 1982).

A Figura 2 mostra a representação gráfica proporcionada pelos softwares Ucinet e Pajek, em conjunto com os fatores determinados pela análise fatorial (Fator 1 representado em amarelo; Fator 2 representado em vermelho; Fator 3 representado em verde).

Figura 2 - Mapa de relações entre os fatores - softwares Ucinet e Pajek. 


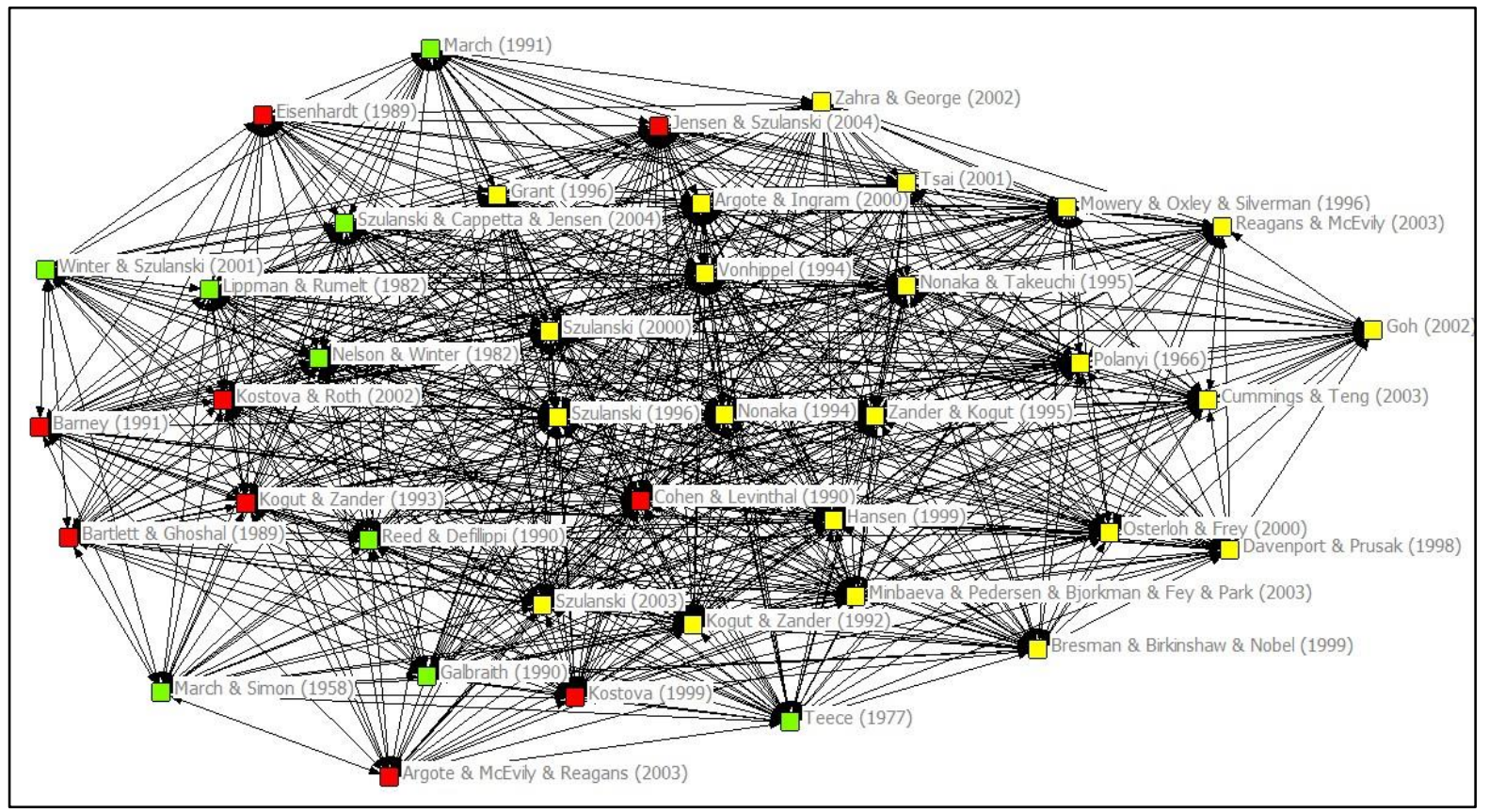

Fonte: Elaborado pelos autores - softwares Ucinet e Pajek.

Os resultados mostram alguns aspectos interessantes. Pode-se verificar que apesar de existir uma relação entre os fatores, o Fator 1 (amarelo) é o predominante onde os autores pesquisam o entender da transferência de conhecimento, mas relacionando parcialmente com os outros fatores. Fator 2 (vermelho) e Fator $\mathbf{3}$ (verde) relacionam-se com mais intensidade comprovando a ligação de transferência de conhecimento dentro das organizações gerando estratégias para inovação.

\section{CONCLUSÃO / CONTRIBUIÇÃO}

O presente estudo teve como objetivo identificar a estrutura intelectual da pesquisa sobre (IS) a partir das referências existentes nas publicações sobre esse tema. Para a realização deste estudo foi usada uma análise bibliométrica de citação e cocitação, em conjunto com a análise fatorial e análise de redes. A característica deste tipo de estudo foi o de mapear a herança intelectual do campo a partir das referências das publicações do tema, o que elucida o mainstream da pesquisa sobre (IS). Com isto, identificou-se 3 campos que apesar de tratarem do mesmo tema (IS), possuem diferenças de linha de estudos. Essas diferenças foram possíveis de serem identificadas com a análise fatorial.

Conclui-se desse estudo, que o primeiro campo, os autores explicam o conceito e tentam entender as suas nuances. Estudam a aprendizagem organizacional, a criação do conhecimento e testam alguns modelos de transferência de conhecimento. Além disso, enfatizam que essa dificuldade de transferir o conhecimento é algo grudento neste processo de transferência. $E$, iniciam uma associação da transferência de conhecimento com a inovação nas empresas. Percebe-se que o conhecimento é visto como residindo dentro do indivíduo, e o papel principal da organização é a aplicação do conhecimento, e não a criação do conhecimento. A capacidade e a motivação são fundamentais para facilitar a transferência de conhecimento nas organizações. A informação grudenta nas empresas é difícil de se compartilhar, onde a informação precisa contribuí para a solução de problemas técnicos e, é de alto valor para adquirir, transferir e ser utilizada em outro departamento ou unidade. O grau de codificação e a facilidade com que as capacidades são ensinadas estão relacionados a velocidade de transferência. Quando o novo conhecimento é desenvolvido por alguém, as empresas encarregamse em articular e ampliar desse conhecimento. O processo de transferência de conhecimento apresenta estágios de transferência e fatores que podem ter ligação com a dificuldade da transferência. Diante disso, é importante examinar empiricamente a natureza e as fontes das barreiras da transferência de melhores práticas dentro das organizações. E, as organizações devem ser baseadas no compartilhamento, ou seja, na transferência de 
conhecimento entre indivíduos e grupos de uma organização.

Do primeiro campo de estudos para o segundo, pôde-se perceber uma evolução nas discussões, onde as questões dos benefícios positivos da transferência de conhecimento para as organizações estavam mais presentes. Dessa forma, surge, o segundo campo de estudos. Os autores, nitidamente, discutem sobre a relação da transferência de conhecimento e a eficiência da organização, as práticas organizacionais, o contexto organizacional junto com a inovação. Fatores como o perfil e contexto relacional dentro das organizações podem aumentar o grau de confiança de uma fonte de conhecimento, influenciando de forma positiva a transferência de prática organizacional. As práticas organizacionais são fundamentais para as multinacionais elevarem o conhecimento na busca de vantagem competitiva.

Por fim o terceiro campo, faz essa transição para as discussões mais voltadas para a inovação estratégica, e aprendizagem para a inovação das empresas. Os autores abordam a transferência de conhecimento com uma argumentação mais voltada para a estratégia da empresa e seu retorno econômico com a transferência de conhecimento. Apresentam os pontos de uma teoria da estratégia de replicação. A estratégia de replicação colabora no processo de alavancar ativos de conhecimento. A interação do tema da transferência de conhecimento com a estratégia, resgata em estudos sobre a Visão Baseada em Conhecimento, a Visão Baseada em Recursos e a Ambiguidade Causal.

Na busca realizada foi possível identificar que não tinha nenhum artigo de revisão sobre o tema (IS). Então, este estudo pôde contribuir com a teoria e mostrar os trabalhos existentes que tiveram uma maior influência sobre (IS). Percebe-se que os artigos existentes tratam de (IS) como a dificuldade de transferência de conhecimento. Com isto, este estudo pode contribuí também com a prática da transferência de conhecimento, principalmente, porque este tema está relacionado com o desenvolvimento da vantagem competitiva nas empresas.

Como limitação do estudo, pode-se apresentar uma limitação tradicional dos estudos bibliométricos que é a escolha de palavras-chave para busca. Esta limitação se deve por ter sido usada diretamente a palavra-chave 'Stickiness". Outra limitação é a escolha da base ISI Web of Science, embora considerada a principal base de artigos acadêmicos, existem outras bases que poderiam ser pesquisadas. Este estudo é o início de uma pesquisa sobre o tema (IS). A intenção é a de dar continuação a pesquisa com um estudo bibliométrico de pareamento. Com o estudo de pareamento será possível identificar as tendências da pesquisa na área, compreendendo o que os autores estão discutindo sobre o tema atualmente. Isso, contribuirá para identificar as lacunas de pesquisas sobre (IS) e possibilitará a continuidade do projeto de pesquisa sobre esse tema.

\section{REFERÊNCIAS}

Acedo, F. J., Barroso, C., \& Galan, J. L. (2006). The resource-based theory: dissemination and main trends. Strategic Management Journal, 27(7), 621636. doi: 10.1002/smj.532

Adler, P. S. (1990). Shared Learning. Management Science, 36(8), 938-957. doi: 10.1287/mnsc.36.8.938

Akbar, H. (2003). Knowledge Levels and their Transformation: Towards the Integration of Knowledge Creation and Individual Learning*. Journal of Management Studies, 40(8), 1997-2021. doi: 10.1046/j.1467-6486.2003.00409.x

Andrews, K. M., \& Delahaye, B. L. (2000). Influences on Knowledge processes In Organizational Learning: The Psychosocial Filter. Journal of Management Studies, 37(6), 797-810. doi: 10.1111/1467-6486.00204

Argote L (2012) Organizational Learning: Creating, Retaining and Transferring Knowledge (Springer Science and Business Media, New York).

Argote, L., \& Ingram, P. (2000). Knowledge Transfer: A Basis for Competitive Advantage in Firms. Organizational Behavior and Human Decision Processes, 82(1), 150-169. doi: 10.1006/obhd.2000.2893

Argote, L., McEvily, B., \& Reagans, R. (2003). Managing Knowledge in Organizations: An Integrative Framework and Review of Emerging Themes. Management Science, 49(4), 571-582. doi: 10.1287/mnsc.49.4.571.14424 
Balm, G. J. (1992). Benchmarking: A Practitioner's Guide for Becoming and Staying Best of the Best.' QPMA Press, Schaumberg, IL.

Baloff, N. (1970). Startup management. IEEE Transactions on Engineering Management, EM-17(4), 132-141. doi: 10.1109/TEM.1970.6448538

Barney, J., (1991). Firm Resources and Sustained Competitive Advantage. Journal of Management. 17(1), 99-120.

Bartlett, C. A., \& Ghoshal, S. (1989). Managing Across Borders: The Transnational Solution. Harvard Business School Press.

Berger, P. L. and T. Luckman (1966). The Social Construction of Reality: A Treatise in the Sociology of Knowledge. Doubleday, Garden City, NY.

Bresman, H., Birkinshaw, J., \& Nobel, R. (1999). Knowledge Transfer In International Acquisitions. Journal of International Business Studies, 30 (3), 439462.

Buttolph, D. (1992). A New Look at Adaptation. Knowledge, 13(4), 460-470. doi: 10.1177/107554709201300405

Chew, W. 8. (1991). 'Productivity, investment and Murphy's law', Harvard Business School, working paper, pp. 91-059.

Cohen, W. M., \& Levinthal, D. A. (1990). Absorptive Capacity: A New Perspective on Learning and Innovation. Administrative Science Quarterly, 35(1), 128. doi: 10.2307/2393553

Cummings, J. L., \& Teng, B.-S. (2003). Transferring R\&D knowledge: the key factors affecting knowledge transfer success. Journal of Engineering and Technology Management, 20(1-2), 39-68. doi: 10.1016/S0923-4748(03)00004-3

Das, A., \& Chakraborty, S. (2018). KNOWLEDGE WITHHOLDING WITHIN AN ORGANIZATION: THE PSYCHOLOGICAL RESISTANCE TO KNOWLEDGE SHARING LINKING WITH TERRITORIALITY. Journal on Innovation and Sustainability. RISUS ISSN 2179-3565, 9, 94. doi: 10.24212/2179-3565.2018v9i3p94-108
Davenport, T. H. \& Prusak, L. (1998). Working knowledge: how organizations manage what they know. Harvard Business School Press. Boston, Massachusetts.

Dierickx, I., \& Cool, K. (1989). Asset Stock Accumulation and Sustainability of Competitive Advantage. Management Science, 35(12), 15041511. doi: $10.1287 / \mathrm{mnsc} .35 .12 .1504$

Eisenhardt, K. M. (1989). Building Theories from Case Study Research. The Academy of Management Review, 14(4), 532. doi: 10.2307/258557

Fabrigar, L. R., Wegener, D. T., MacCallum, R. C., \& Strahan, E. J. (1999). Evaluating the Use of Exploratory Factor Analysis in Psychological Research. 28.

Galbraith, C. S. (1990). Transferring Core Manufacturing Technologies in High-Technology Firms. California Management Review, 32(4), 56-70. doi: $10.2307 / 41166628$

Goh, S. C. (2002). Managing effective knowledge transfer: an integrative framework and some practice implications. Journal of Knowledge Management, 6(1), 23-30. doi: 10.1108/13673270210417664

Grant, R. M. (1996). Toward a knowledge-based theory of the firm. Strategic Management J. 17 109122.

Guerrazzi, L. A. de C., Brandão, M. M., Junior, H. de C., \& Lourenço, C. E. (2015). Pesquisa em Marketing e Estratégia nos Principais Periódicos Internacionais: Um Estudo Bibliométrico sobre Publicações no Século XXI. Revista Ibero-Americana de Estratégia, 14(01), 07-27. doi: 10.5585/riae.v14i1.2200

Gupta, A. K., \& Govindarajan, V. (2000). Knowledge flows within multinational corporations. Strategic Management Journal, 21(4), 473-496. Retrieved December 18, 2020, from http://www.jstor.org/stable/3094239

Hansen, M. (1999). The search-transfer problem: The role of weak ties in sharing knowledge across organization subunits. Administrative Science Quarterly, 44(1) 82-111. 
Hayes, R. H. and K. B. Clark (1985). Exploring the Sources of Productivity Differences at the Factory Level. Wiley, New York.

Jasimuddin, S. M. (2007). Exploring knowledge transfer mechanisms: The case of a UK-based group within a high-tech global corporation. International Journal of Information Management, 27(4), 294-300. doi: 10.1016/j.ijinfomgt.2007.03.003

Jensen, R., \& Szulanski, G. (2004). Stickiness and the adaptation of organizational practices in crossborder knowledge transfers. Journal of International Business Studies, 35(6), 508-523. doi: 10.1057/palgrave.jibs. 8400107

Kogut, B., \& Zander, U. (1992). Knowledge of the firm, combinative capabilities and the replication of technology. Organization Science, 3(3), 383-397.

Kogut, B., \& Zander, U. (1993). KNOWLEDGE OF THE FIRM AND THE EVOLUTIONARY THEORY OF THE MULTINATIONAL CORPORATION. JOURNAL OF INTERNATIONALBUSINESS STUDIES, 24 (4), 625-645.

Kostova, T. (1999). Transnational Transfer of Strategic Organizational Practices: A Contextual Perspective. The Academy of Management Review, 24(2), 308. doi: $10.2307 / 259084$

Lane, P. J., \& Lubatkin, M. (1998). Relative absorptive capacity and interorganizational learning. Strategic Management Journal. 19:461-477.

Leonard-Barton, D. A., W. B. Chew, \& R. Bohn. (1991). "Beating Murphy's Law." MIT Sloan Management Review 32, no. 3: 5-16.

Lin, T.-Y., \& Cheng, Y.-Y. (2010). EXPLORING THE KNOWLEDGE NETWORK OF STRATEGIC ALLIANCE RESEARCH: A CO-CITATION ANALYSIS. 8(2), 9.

Lippman, S. A., \& Rumelt, R. P. (1982). Uncertain Imitability: An Analysis of Interfirm Differences in Efficiency under Competition. The Bell Journal of Economics, 13(2), 418. doi: 10.2307/3003464

March, J. and H. Simon (1958). Organizations. Wiley, New York.
March, J. G. (1991). Exploration and exploitation in organizational learning. Organ. Sci. 2(1) 71-87.

McCain, K. W. (1990). Mapping authors in intellectual space: A technical overview. Journal of the American Society for Information Science, 41(6), 433-443.

Minbaeva, D., Pedersen, T., Björkman, I, Fey, C. F., \& Park, H. J. (2003). MNC knowledge transfer, subsidiary absorptive capacity and HRM. Journal of International Business Studies, 34, 586-59.

Mowery, D. C., Oxley, J. E., \& Silverman, B. S. (1996). Strategic alliances and interfirm knowledge transfer: Strategic Alliances and Interfirm Knowledge Transfer. Strategic Management Journal, 17(S2), 7791. doi: 10.1002/smj.4250171108

Nath, R., \& Jackson, W. M. (1991). Productivity of management information systems researchers: Does Lotka's law apply? Information Processing \& Management, 27(2-3), 203-209. doi: 10.1016/03064573(91)90049-R

Nelson, R. and S. Winter (1982). An Evolutionary Theory of Economic Change. Belknap Press, Cambridge, MA.

Nonaka, I. (1994). A dynamic theory of organizational knowledge creation. Organization Science, 5, pp. 14-37.

Nonaka, I., Takeuchi, H. (1995). The KnowledgeCreating Company. Oxford University Press, New York and Oxford, UK.

Osterloh, M., \& Frey, B. S. (2000). Motivation, Knowledge Transfer, and Organizational Forms. Organization Science, 11(5), 538-550. doi: 10.1287/orsc.11.5.538.15204

Pilkington, A., \& Meredith, J. (2009). The evolution of the intellectual structure of operations management-1980-2006: A citation/co-citation analysis. Journal of Operations Management, 27(3), 185-202. doi: 10.1016/j.jom.2008.08.001

Polanyi, M. (1966). The Tacit Dimension. Routledge and Kegan Paul, London, UK. 
Ramos-Rodríguez, A.-R., \& Ruíz-Navarro, J. (2004). Changes in the intellectual structure of strategic management research: a bibliometric study of theStrategic Management Journal, 1980-2000. Strategic Management Journal, 25(10), 981-1004. doi: 10.1002/smj.397

Reagans, R., \& McEvily, B. (2003). Network Structure and Knowledge Transfer: The Effects of Cohesion and Range. Administrative Science Quarterly, 48(2), 240. doi: 10.2307/3556658

Reed, R., \& Defillippi, R. J. (1990). Causal Ambiguity, Barriers to Imitation, and Sustainable Competitive Advantage. The Academy of Management Review, 15(1), 88. doi: 10.2307/258107

Rice, R. E. and E. M. Rogers (1980). 'Reinvention in the innovation process'. Knowledge: Creation, Diffusion, Utilization, 1(4), pp. 499-514.

Schuller, M. (2014). Stickiness in knowledge transfer. In H. Hasan (Eds.), Being Practical with Theory: A Window into Business Research (pp. 6163). Wollongong, Australia: THEORI. https://eurekaconnection.files.wordpress.com/2014 /02/p-61-63-stickiness-in-knowledge-theory-theoriebook_finaljan2014-v3.pdf

Serra, F., Ferreira, M., Guerrazzi, L., \& Scaciotta, V. (2018). Doing Bibliometric Reviews for the Iberoamerican Journal of Strategic Management. Iberoamerican Journal of Strategic Management (IJSM), 17(3), 01-16. doi:10.5585/riae.v17i3.2713.

Shafique, M. (2013). Thinking inside the box? Intellectual structure of the knowledge base of innovation research (1988-2008): Intellectual Structure of Innovation Research (1988-2008). Strategic Management Journal, 34(1), 62-93. doi: 10.1002/smj.2002

Simonin, B. L. (1999). Ambiguity and the process of knowledge transfer in strategic alliances. Strategic Management Journal, 20(7), 595-623. Retrieved December 18, 2020, from http://www.jstor.org/stable/3094131

Small, H. (1973). Co-citation in the scientific literature: $A$ new measure of the relationship between two documents. Journal of the American
Society for Information Science, 24(4), 265-269. doi: 10.1002/asi.4630240406

Szulanski, G. (1996). Exploring internal stickiness: Impediments to the transfer of best practice within the firm: Exploring Internal Stickiness. Strategic Management Journal, 17(S2), 27-43. doi: 10.1002/smj.4250171105

Szulanski, G. (2000). The Process of Knowledge Transfer: A Diachronic Analysis of Stickiness. Organizational Behavior and Human Decision Processes, 82(1), 9-27. doi: 10.1006/obhd.2000.2884

Szulanski, G. (2003). Sticky knowledge: Barriers to knowing in the firm. London: Sage.

Szulanski, G., Cappetta, R., \& Jensen, R. J. (2004). When and How Trustworthiness Matters: Knowledge Transfer and the Moderating Effect of Causal Ambiguity. Organization Science, 15(5), 600-613. doi: 10.1287/orsc.1040.0096

Szulanski, G., Ringov, D., \& Jensen, R. J. (2016). Overcoming Stickiness: How the Timing of Knowledge Transfer Methods Affects Transfer Difficulty. Organization Science, 27(2), 304-322. doi: 10.1287/orsc.2016.1049

Tallman, S., Jenkins, M., Henry, N., \& Pinch, S. (2004). Knowledge, Clusters, and Competitive Advantage. The Academy of Management Review, 29(2), 258. doi: $10.2307 / 20159032$

Teece, D. J. (1977). Technology Transfer by Multinational Firms: The Resource Cost of Transferring Technological Know-How. The Economic Journal, 87(346), 242. doi: 10.2307/2232084

Tolbert, P. S. (1988). Institutional sources of organizational culture in major law firms [Electronic version]. In L. G. Zucker (Ed.), Institutional patterns and organizations: Culture and environment (pp. 101113). Boston: Ballinger Press.

Tsai, W. (2001). Knowledge transfer in intraorganizational networks: Effects of network position and absorptive capacity on business unit innovation and performance. Academy of Management Journal, 44(5), 996-1004. doi: $10.2307 / 3069443$ 
Tyre, M. J., \& Orlikowski, W. J. (1994). Windows of Opportunity: Temporal Patterns of Technological Adaptation in Organizations. Organization Science, 5(1), 98-118. doi: 10.1287/orsc.5.1.98

Urbizagástegui Alvarado, R. (2002). A Lei de Lotka na bibliometria brasileira. Ciência da Informação, 31(2), 14-20. doi: 10.1590/S010019652002000200002

Vogel, R., \& Güttel, W. H. (2012). The Dynamic Capability View in Strategic Management: A Bibliometric Review: DCV in Strategic Management. International Journal of Management Reviews, n/an/a. doi: 10.1111/ijmr.12000

von Hippel, E. (1994). "Sticky Information" and the Locus of Problem Solving: Implications for Innovation. Management Science, 40(4), 429-439. doi: 10.1287/mnsc.40.4.429

Wang, Z., \& Wang, N. (2012). Knowledge sharing, innovation and firm performance. Expert systems with applications, 39(10), 8899-8908.
Winter, S. G., \& Szulanski, G. (2001). Replication as Strategy. Organization Science, 12(6), 730-743. doi: 10.1287/orsc.12.6.730.10084

Zahra, S. A., \& George, G. (2002). Absorptive capacity: a review, reconceptualization, and extension. Academy of Management Review. 27 (2), 185-203.

Zaltman. G., R. Duncan and J. Holbek (1973). Innovations and Organizations. Wiley, New York.

Zander, U., \& Kogut, B. (1995). Knowledge and the speed of the transfer and imitation of organizational capabilities: An empirical test. Organization Science, 6(1) 76-92.

Zupic, I., \& Čater, T. (2015). Bibliometric Methods in Management and Organization. Organizational Research Methods, 18(3), 429-472. doi: 10.1177/1094428114562629 